\title{
A Novel Hybrid Multi-objective BB-BC based Channel Allocation Algorithm to Reduce FWM Crosstalk and its Comparative Study
}

\author{
Suruchi Bali \\ Department of Electronics and \\ Communication Engineering \\ Seth Jai Parkash Mukand Lal \\ Institute of Engineering and \\ Technology, Radaur, India
}

\author{
Shonak Bansal \\ Department of Electronics and \\ Communication Engineering \\ PEC University of Technology, \\ Sector-12, Chandigarh, India
}

\author{
Anil Kamboj \\ Department of Electronics and \\ Communication Engineering \\ Seth Jai Parkash Mukand Lal \\ Institute of Engineering and \\ Technology, Radaur, India
}

\begin{abstract}
Nature is a good source of inspirations for us. The algorithms developed from the nature are most powerful algorithms for optimizing many complex engineering design problems having multiple objectives (multi-objective). This paper presents an hybrid algorithm based on Multi-objective Big bang-Big Crunch (MOBB-BC) nature-inspired optimization algorithm with Genetic crossover and Differential evolution (DE) mutation operators for solving the minimum length ruler called Optimal Golomb ruler (OGR) as channel-allocation problem to reduce four-wave mixing crosstalk (FWM) effects in optical wavelength division multiplexing (WDM) systems. The comparative study of simulation results obtained by proposed hybrid Multi-objective BB-BC (HMOBB-BC) algorithm demonstrates better and efficient generation of OGRs in a reasonable computational time compared to simple $\mathrm{BB}-\mathrm{BC}$ algorithm and one of the existing nature-inspired algorithms i.e. Genetic algorithm (GA). Also, the proposed hybrid algorithm outperforms the two existing conventional algorithms i.e. Extended quadratic congruence (EQC) and Search algorithm (SA), in terms of ruler length and total channel bandwidth.
\end{abstract}

\section{General Terms}

Conventional computing, Four-wave mixing, Multiobjective, Nature-inspired, Optimization.

\section{Keywords}

Channel spacing, Genetic algorithm, Hybrid Multi-objective Big bang-Big Crunch optimization algorithm, Optimal Golomb ruler.

\section{INTRODUCTION}

Crosstalk due to four-wave mixing (FWM) is the dominant nonlinear effect in a multi-channel long haul optical communication fiber system which limits the performance of optical wave length division multiplexing (WDM) system. It is therefore important to develop algorithms to allocate the channel frequencies in order to reduce the FWM crosstalk [1]-[6].

There is several unequally spaced channel-allocation algorithms have been proposed [2], [7]-[14] which have the drawback of increased optical channel bandwidth requirement compared to equally spaced channel allocation. This paper proposes a nature-inspired based unequally spaced channel algorithm by using the concept of Optimal Golomb ruler (OGR) sequences [15]-[17].

By using OGRs as channel-allocation, reduction in FWM crosstalk can be achieved in the optical WDM systems without affecting total optical channel bandwidth. Golomb rulers represent a class of $N P$-complete [18] problem. Several different algorithms are proposed to solve Golomb ruler problem such as exact methods [19], [20], constraint programming [21], local searches [22] and exhaustive parallel search [23]. There are various nature-inspired based algorithms such as Genetic algorithm (GA) [24]-[28], Biogeography Based Optimization (BBO) [28]-[30], Big Bang-Big Crunch (BB-BC) algorithm [31], [32], Firefly algorithm (FA) [33], Cuckoo search based algorithm (CSA) [34], and Multi-objective flower pollination algorithm (MOFPA) and its hybridization form [35] to solve the OGRs problem. This paper introduces two concepts i.e. hybridization and multi-objective in simple BB-BC optimization algorithm to solve unequally spaced channel-allocation problem in optical WDM system. The hybridization of BB-BC algorithm is done with Genetic crossover and Differential Evolution mutation operator. The purpose of hybridization is to improve the convergence rate and precision of $\mathrm{BB}-\mathrm{BC}$ algorithm. Then formed hybrid BB-BC algorithm is extended to multiobjective optimization problems by using a Pareto-based approach [36], [37]. Both these concepts are combined in order to generate OGR sequences for various marks or optical WDM's channels.

This paper has following sections: Section 2 introduces the brief concept of Golomb rulers. Section 3 introduces with hybrid Multi-objective BB-BC nature-inspired optimization algorithm. Section 4 presents the problem formulation. Section 5 presents the simulation results and performance comparison of proposed algorithm and Section 6 presents the conclusion and future scope of the research.

\section{GOLOMB RULERS}

Golomb ruler refers to a set of positive integers named as marks and no distinct pairs of numbers from the set have the same difference [38]-[40]. The difference between the values of any two marks is called the distance between those marks. The difference between the largest and smallest number is referred to as the length of the ruler. The number of marks on a ruler is referred to as the size of the ruler. 


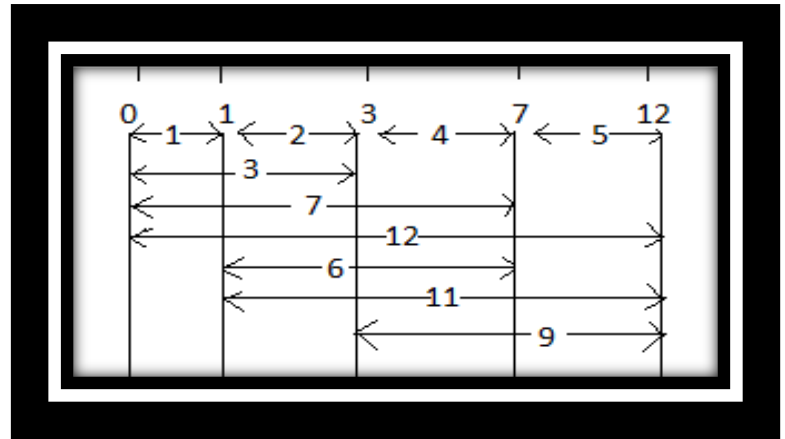

Fig1: A 5-Marks non-OGR having Ruler Length 12with its associated distances

A perfect Golomb ruler measures all the non-negative integer distances from 0 to length $L$ of the ruler [40]-[43]. An optimal Golomb ruler is the shortest length ruler for a given mark. There can be numerous different OGRs for a specific marks value. Figure 1 show an example of 5-marks non- optimal Golomb ruler having ruler length 12 . The distance associated between each pair of marks is also shown in Figure 1. As clear from Figure 1 that the distance numbers 8 and 10 are missing so it is not a perfect Golomb ruler sequence.

\section{HYBRID MULTI-OBJECTIVE BIG BANG-BIG CRUNCH ALGORITHM}

Big Bang-Big Crunch algorithm, a meta-heuristic population-based optimization algorithm relies on the theories of the evolution of universe; called the Big Bang and Big Crunch theory [44]-[46]. It states us that the Universe's expansion is due to the Big Bang and will not continue forever. Instead, at a certain point in time, it will stop expanding and collapse into itself, pulling everything with it until it eventually turns into the biggest black hole ever. Erol and Eksin [47], inspired by these theories, introduced an optimization algorithm named Big Bang-Big Crunch optimization algorithm. This algorithm has two phases: the first phase is Big Bang phase and the second phase is Big Crunch phase where a contraction procedure calculates a center of mass for the population [48], [49]. These two phases represent the best solution exploitation and large search space exploration, respectively. The first phase (energy dissipation) randomly generates an initial population of feasible candidate solutions. Generally, this phase represents the search space exploration process. After the Big Bang phase, a contraction procedure is applied during the Big Crunch. This aims to have quick convergence and reduce computational time, while maintaining the quality of solutions and search diversity. The best candidate solution is represented as the centre of mass that will attract other solutions. In the Big Crunch phase, the contraction operator takes the current positions of each candidate solution in the population and its associated fitness/cost function value and computes a centre of mass according to the equation (1) [47]:

$$
x_{c}=\frac{\sum_{i=1}^{\text {Popsize }} \frac{1}{f_{i}} x_{i}}{\sum_{i=1}^{\text {Popsize }} \frac{1}{f_{i}}}
$$

where, $x_{c}$ is position of the centre of mass, $x_{i}$ is position of the candidate, $f_{i}$ is fitness (cost) function value of the candidate $i$; and Popsize is the population size.

Instead of the centre of mass, best fit individual can also be chosen as the starting point in the Big Bang phase. The new candidates $\left(x_{\text {new }}\right)$ around the centre of mass are calculated by subtracting or adding a normal random number whose value decreases as the iterations elapse. This can be formalized by the equation (2):

$$
x_{\text {new }}=x_{c}+r \times c_{1} \times \frac{\left(x_{\max }-x_{\min }\right)}{1+t / c_{2}}
$$

where $r$ is a standard normal distribution random number, $c_{l}$ is a limiting the size parameter of the search space, parameter $c_{2}$ denotes after how much iteration the search space will be restricted to half, $x_{\max }$ and $x_{\min }$ are the upper and lower limits, and $t$ is the iteration index.

Although the algorithm, BB-BC has exceptional property as compared to numerous nature-inspired optimization algorithms while solving lower-dimensional optimization design problems, but may become challenging for higherdimensional optimization design problems because of the phenomenon of slow convergence and low accuracy rates. This means there are some problems in the global exploitation and exploration of the search space.

Therefore, this paper forward an improved hybrid BB-BC with multiple objectives, namely, HMOBB-BC, that relies on Genetic crossover [50] and fitness values based differential mutation strategy [51], [52] to accelerate the convergence speed of multi-objective BB-BC (MOBB-BC) algorithm. A multi-objective optimization problem with $M$ objectives can be written in general as [37]:

$$
\text { Maximize/Minimize } f_{1}(x), f_{2}(x), \ldots, f_{M}(x)
$$

subject to the non-linear equality and inequality constraints.

$$
h_{j}(x)=0,(j=1,2, \ldots, J) \text { and } g_{k}(x) \geq 0,(k=1,2, \ldots, K) .
$$

One of the simplest ways is to use a weighted sum to combine multi-objective into a composite single objective is given by equation (3) [37]:

$$
\begin{aligned}
& \text { with } \sum_{i=1}^{M} w_{i=1}^{M} w_{m} f_{m}, \quad w_{i}>0
\end{aligned}
$$

where $w_{i}(i=1, \ldots, M)$ are randomly generated non-negative weights.

The fundamental idea of this weighted sum approach is that these weighting coefficients act as the preferences for these multi-objectives [37].

In proposed HMOBB-BC, algorithm the mutation rate probability $M R_{i}^{t}$ of each solution $x_{i}$ at running iteration index $t$ is determined based on the fitness value $f_{i}^{t}$ of each solution:

$$
M R_{i}^{t}=\frac{f_{i}^{t}}{\operatorname{Max}\left(f^{t}\right)}
$$

where $\operatorname{Max}\left(f^{t}\right)$ is maximum fitness value in the population of solutions at iteration $t$.

In order to improve the search efficiency and increase the population diversity, based on the mutation rate probability the positions of the candidates $x_{i}$ (solutions) are updated by using the "DE/rand/1" [52] mutation equation (6):

$$
x_{i}^{t}=x_{r_{1}}^{t-1}+P_{\text {mutate }}\left(x_{r_{2}}^{t-1}-x_{r_{3}}^{t-1}\right)
$$




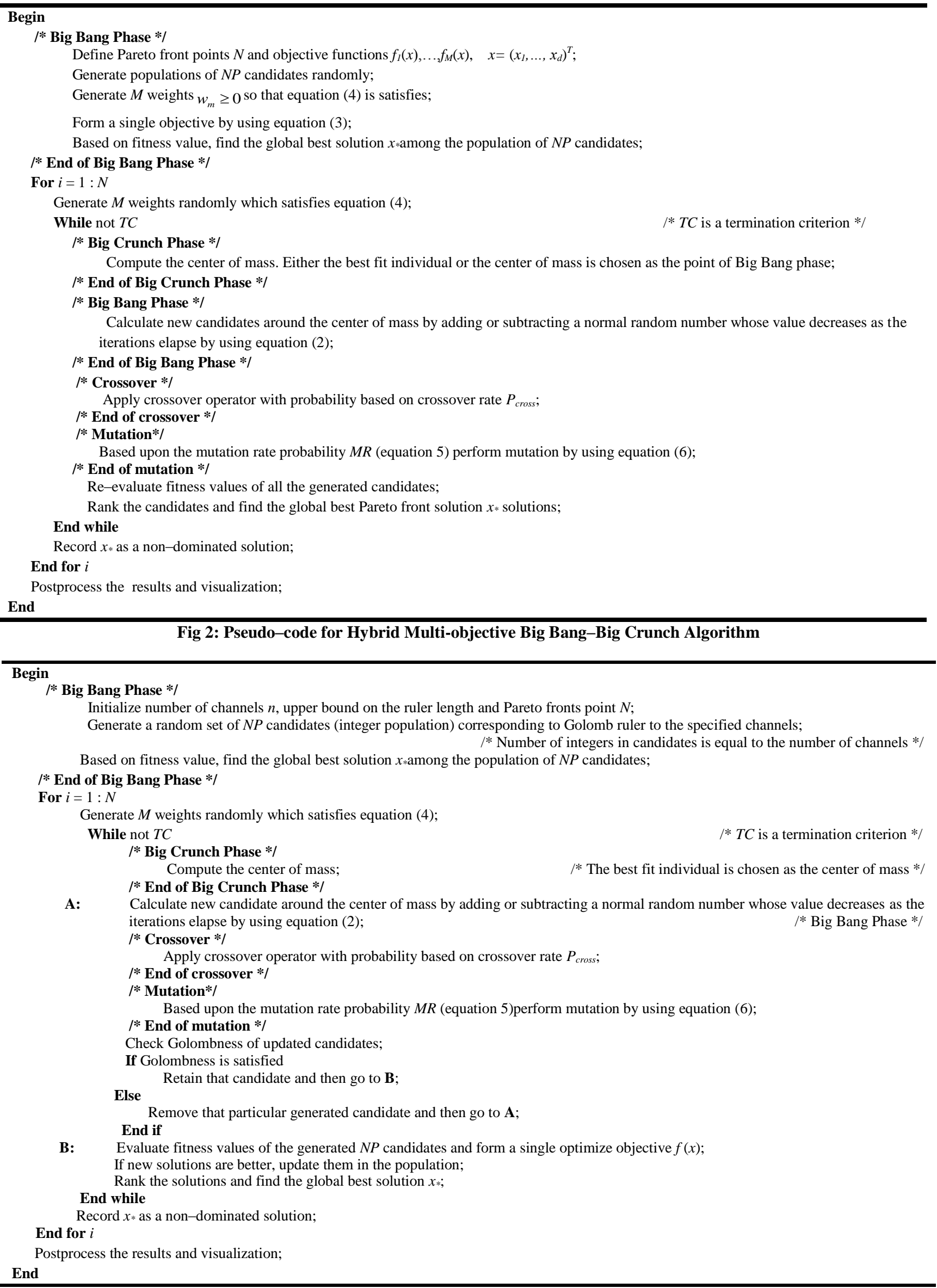

Fig 3: Pseudo-code for HMOBB-BC Algorithm to Generate OGR Sequences 
where $r_{1}, r_{2}$ and $r_{3}$ are randomly chosen mutually different integers from the interval $[0, N P-1]$ and $P_{\text {mutate }}>0$ is the mutation rate. The integers $r_{1}, r_{2}$ and $r_{3}$ are different from the running index $i$. $P_{\text {mutate }}$ is a real and constant factor which controls the amplification of the differential variation. The differential mutation strategy increases the chances for a good solution, but a high mutation rate $(>1)$ results in too much exploration and is disadvantageous to the improvement of candidate solutions [28]. Based upon the above discussion, the corresponding pseudo code for HMOBB-BC algorithm is shown in Figure 2.

\section{PROBLEM FORMULATION}

If the spacing between any pair of channels is denoted as $C S$ and the total number of channels is $n$, then the objective is to optimize the length of the ruler denoted as $R L$, which is given by the equation (7) [28]:

$$
R L=\sum_{i=1}^{n-1}(C S)_{i}
$$

subject to $(C S)_{i} \neq(C S)_{j}$.

If each individual element is a Golomb ruler, the sum of all elements of an individual forms the total optical bandwidth of the channels. Thus, if an individual element is denoted as $I E$ then the second objective is to minimize the total optical bandwidth $T B W$ which is given by the equation (8):

$$
\begin{aligned}
& T B W=\sum_{i=1}^{n}(I E)_{i} \\
& \text { subject to }(I E)_{i} \neq(I E)_{j} .
\end{aligned}
$$

where $i, j=1,2, \ldots, n$ with $i \neq j$ are distinct in both equations (7) and (8).

The proposed pseudo-code for HMOBB-BC algorithm to generate OGR sequences as unequally spaced channelallocation in optical WDM system is shown in Figure 3.

\section{SIMULATION RESULTS AND DISCUSSION}

To find unequal spaced channel-allocation algorithm in optical WDM systems i.e. OGR sequences, the proposed HMOBB-BC algorithm has been written and verified in Matlab-7 language [53] under Windows 7 operating system. To show the effectiveness of the proposed algorithm, its performance is being compared with known OGRs [15], [20], [38]-[42], [54]-[56], EQC, SA [2], [13], [24], GA [28], and BB-BC [31], [32] algorithms of generating unequal spaced WDM channel-allocation sequences.

\subsection{Simulation Parameters for Hybrid Multi-objective Big Bang-Big Crunch}

To generate optimal Golomb ruler sequences as optical WDM channel-allocation, after a number of careful experimentation, the optimum values of HMOBB-BC parameters finally been settled in this research is reported in Table 1.

It is noted that the iterations has little effect for low order marks for examples $n=3$ and 4 . But for higher order marks, the iterations has a great effect on the performance of HMOBB-BC algorithm i.e. ruler length and total bandwidth gets optimized after a certain numbers of iterations. As the number of iterations increases, the length of the ruler and hence the total optical bandwidth of the sequence tends to decrease; it means that the rulers reach their optimum values after a certain number of iterations. This is the point where the
Table 1. Simulation parameters for HMOBB-BC Algorithm

\begin{tabular}{l|l}
\hline \multicolumn{1}{c|}{ Parameter } & \multicolumn{1}{c}{ Value } \\
\hline$c_{1}$ & 0.1 \\
$c_{2}$ & 5 \\
Number of candidates (Popsize) & 20 \\
Crossover method & Single Point \\
Crossover probability $\left(P_{\text {cross }}\right)$ & 1 \\
Mutation rate $\left(P_{\text {mutate }}\right)$ & 0.05 \\
Iterations & 1000 \\
\hline
\end{tabular}

results are optimum and no further improvement is seen, that is, we are approaching towards the optimal solution. By carefully observation, the paper fixed the iterations of 1000 for HMOBB$\mathrm{BC}$ algorithm. With these parameters values, a number of sets of trials for various order marks are conducted.

\subsection{Comparison of HMOBB-BC Algorithm with Previous Existing Algorithms in Terms of Ruler Length, Total Bandwidth and Average CPU Time}

The purpose to use HMOBB-BC algorithm in this paper is to optimize the length of the ruler so as to conserve the total bandwidth occupied by the channels in less computational time. Table 2 list the length of ruler $(R L)$, total optical bandwidth $(T B W)$ and average CPU time occupied by different sequences obtained by proposed algorithm for various channels $n$ and its comparison with known OGRs [15], [20], [38]-[42], [54]-[56], EQC, SA [2], [13], [24], GA [28], and BB-BC [31], [32] algorithms.

The application of conventional algorithms i.e. EQC and SA is limited to prime powers [2], so the length of ruler and total bandwidth for EQC and SA are shown by a dash line in Table 2. Comparing the simulation results obtained from $\mathrm{HMOBB}-$ $\mathrm{BC}$ algorithm with known OGRs, EQC, SA, GA and BB-BC; it is perceived that there is a significant improvement with respect to the length of the ruler, the total bandwidth occupied and average CPU time that is, the results gets better. Figure 4 (a) and 4 (b) illustrates the graphical comparison of HMOBB$\mathrm{BC}$ algorithm to generate OGR sequences for optical WDM system with existing algorithms in terms of the length of the ruler and total optical bandwidth occupied by the various order mark values respectively, whereas Figure 5 illustrates the comparison of proposed HMOBB-BC algorithm with GA and $\mathrm{BB}-\mathrm{BC}$ algorithm in terms of average CPU time (in Sec.) for various order marks. So, it is concluded from Table 2, Figures 4 and 5 that the performance of proposed HMOBB$\mathrm{BC}$ algorithm is better than the existing algorithms.

\section{CONCLUSION}

This paper presented the application of nature-inspired multiobjective BB-BC optimization algorithm and its hybridization with Genetic crossover and differential mutation operator to find optimal Golomb ruler sequences needed for optical WDM systems. The optimal Golomb ruler's sequence provides the unequal channel-allocation in optical WDM systems to reduce the FWM crosstalk. It has been observed that proposed HMOBB-BC algorithm produces Golomb ruler sequences very efficiently and effectively. The performance is being compared with the existing conventional and natureinspired algorithms in terms of the length of ruler, total optical channel bandwidth and average CPU time obtained by the 
Table 2: Performance Comparison of proposed HMOBB-BC Algorithm with Known OGR, EQC, SA, GA and BB-BC in terms of Ruler Length, Total Bandwidth, and Average CPU Time

\begin{tabular}{|c|c|c|c|c|c|c|c|c|c|c|c|c|c|c|c|}
\hline \multirow{4}{*}{$n$} & \multirow{3}{*}{\multicolumn{2}{|c|}{$\begin{array}{c}\text { Known OGRs } \\
{[15],[20],} \\
{[38]-[42],} \\
{[54]-[56]}\end{array}$}} & \multicolumn{13}{|c|}{$\begin{array}{c}\text { ALGORITHMS } \\
\end{array}$} \\
\hline & & & \multicolumn{4}{|c|}{ Conventional Algorithms } & \multicolumn{6}{|c|}{ Existing Nature-Inspired Algorithms } & \multirow{2}{*}{\multicolumn{3}{|c|}{$\begin{array}{c}\text { Proposed Algorithm } \\
\text { HMOBB-BC }\end{array}$}} \\
\hline & & & \multicolumn{2}{|c|}{$\begin{array}{c}\mathbf{E Q C} \\
{[2],[13]} \\
{[24]}\end{array}$} & \multicolumn{2}{|c|}{$\begin{array}{c}\text { SA } \\
{[2],[13],} \\
{[24]}\end{array}$} & \multicolumn{3}{|c|}{$\begin{array}{c}\text { GA } \\
{[28]}\end{array}$} & \multicolumn{3}{|c|}{$\begin{array}{c}\text { BB-BC } \\
\text { [31], [32] }\end{array}$} & & & \\
\hline & $R L$ & $\begin{array}{r}T B W \\
(H z)\end{array}$ & $R L$ & $\begin{array}{c}T B W \\
(H z)\end{array}$ & $R L$ & $\begin{array}{c}T B W \\
(H z)\end{array}$ & $R L$ & $\begin{array}{c}T B W \\
(H z)\end{array}$ & $\begin{array}{l}\text { Average } \\
\text { CPU time } \\
\text { (Sec.) }\end{array}$ & $R L$ & $\begin{array}{l}T B W \\
(H z)\end{array}$ & $\begin{array}{l}\text { Average } \\
\text { CPU time } \\
\text { (Sec.) }\end{array}$ & $R L$ & $\begin{array}{c}T B W \\
(H z)\end{array}$ & $\begin{array}{c}\text { Average } \\
\text { CPU time } \\
\text { (Sec.) }\end{array}$ \\
\hline 4 & 6 & 11 & 15 & 28 & 15 & 28 & $\begin{array}{l}6 \\
7\end{array}$ & 11 & 0.001 & $\begin{array}{l}6 \\
7\end{array}$ & 11 & 0.000 & $\begin{array}{l}6 \\
7\end{array}$ & 11 & 0.000 \\
\hline 5 & 11 & $\begin{array}{l}25 \\
28\end{array}$ & - & - & - & - & $\begin{array}{l}12 \\
13\end{array}$ & $\begin{array}{l}23 \\
25 \\
29\end{array}$ & 0.021 & $\begin{array}{l}11 \\
12\end{array}$ & $\begin{array}{l}23 \\
25\end{array}$ & 0.009 & $\begin{array}{l}11 \\
12 \\
13\end{array}$ & $\begin{array}{l}23 \\
25\end{array}$ & 0.001 \\
\hline 6 & 17 & $\begin{array}{l}44 \\
47 \\
50\end{array}$ & 45 & 140 & 20 & 60 & $\begin{array}{l}17 \\
18 \\
21\end{array}$ & $\begin{array}{l}42 \\
44 \\
45\end{array}$ & 0.780 & $\begin{array}{l}17 \\
18\end{array}$ & $\begin{array}{l}42 \\
44\end{array}$ & 0.659 & $\begin{array}{l}17 \\
18\end{array}$ & $\begin{array}{l}42 \\
44\end{array}$ & 0.0539 \\
\hline 7 & 25 & $\begin{array}{l}77 \\
81 \\
87 \\
90 \\
95\end{array}$ & - & - & - & - & $\begin{array}{l}27 \\
28 \\
29 \\
30 \\
31 \\
32\end{array}$ & $\begin{array}{l}73 \\
78 \\
79 \\
80 \\
83 \\
86 \\
95\end{array}$ & 1.120 & $\begin{array}{l}25 \\
26 \\
28 \\
30\end{array}$ & $\begin{array}{l}73 \\
74 \\
77 \\
81\end{array}$ & 1.170 & $\begin{array}{l}25 \\
28\end{array}$ & $\begin{array}{l}74 \\
77 \\
81 \\
90\end{array}$ & 0.0899 \\
\hline 8 & 34 & 117 & 91 & 378 & 49 & 189 & $\begin{array}{l}35 \\
41 \\
42 \\
45 \\
46\end{array}$ & $\begin{array}{l}121 \\
126 \\
128 \\
129 \\
131 \\
133\end{array}$ & 1.241 & $\begin{array}{l}39 \\
41 \\
42\end{array}$ & $\begin{array}{l}113 \\
118 \\
119\end{array}$ & 1.210 & $\begin{array}{l}34 \\
39\end{array}$ & $\begin{array}{l}113 \\
117\end{array}$ & 0.1441 \\
\hline 9 & 44 & 206 & - & - & - & - & $\begin{array}{l}52 \\
56 \\
59 \\
61 \\
63 \\
65\end{array}$ & $\begin{array}{l}192 \\
193 \\
196 \\
203 \\
225\end{array}$ & 1.711 & $\begin{array}{l}44 \\
45 \\
46 \\
61\end{array}$ & $\begin{array}{l}179 \\
248 \\
253 \\
262\end{array}$ & 1.698 & 44 & 206 & 1.1895 \\
\hline 10 & 55 & 249 & - & - & - & - & $\begin{array}{l}75 \\
76\end{array}$ & $\begin{array}{l}283 \\
287 \\
301\end{array}$ & $5.499 \mathrm{e}+01$ & 77 & 258 & $5.450 \mathrm{e}+01$ & 55 & 249 & $3.151 \mathrm{e}+01$ \\
\hline 11 & 72 & $\begin{array}{l}386 \\
391\end{array}$ & - & - & - & - & $\begin{array}{l}94 \\
96\end{array}$ & $\begin{array}{l}395 \\
456\end{array}$ & $7.200 \mathrm{e}+02$ & $\begin{array}{c}72 \\
105\end{array}$ & $\begin{array}{l}377 \\
490 \\
456\end{array}$ & $6.990 \mathrm{e}+02$ & 72 & 386 & $4.765 e+02$ \\
\hline 12 & 85 & 503 & 231 & 1441 & 132 & 682 & $\begin{array}{l}123 \\
128 \\
137\end{array}$ & $\begin{array}{l}532 \\
581 \\
660\end{array}$ & $8.602 \mathrm{e}+02$ & $\begin{array}{l}85 \\
91\end{array}$ & $\begin{array}{l}550 \\
580 \\
613\end{array}$ & $7.981 \mathrm{e}+02$ & 85 & 503 & $5.659 \mathrm{e}+02$ \\
\hline 13 & 106 & 660 & - & - & - & - & $\begin{array}{l}203 \\
241\end{array}$ & $\begin{array}{l}1015 \\
1048\end{array}$ & $1.070 \mathrm{e}+03$ & $\begin{array}{l}110 \\
113\end{array}$ & $\begin{array}{l}768 \\
753\end{array}$ & $1.020 \mathrm{e}+03$ & 106 & 660 & $8.751 \mathrm{e}+02$ \\
\hline 14 & 127 & 924 & 325 & 2340 & 286 & 1820 & $\begin{array}{l}206 \\
228 \\
230\end{array}$ & $\begin{array}{l}1172 \\
1177 \\
1285\end{array}$ & $1.028 \mathrm{e}+03$ & 221 & 1166 & $1.021 \mathrm{e}+03$ & 127 & 924 & $1.013 \mathrm{e}+03$ \\
\hline 15 & 151 & 1047 & - & - & - & - & $\begin{array}{l}275 \\
298\end{array}$ & $\begin{array}{l}1634 \\
1653\end{array}$ & $1.440 \mathrm{e}+03$ & 267 & 1322 & $1.291 \mathrm{e}+03$ & 151 & 1047 & $1.165 \mathrm{e}+03$ \\
\hline 16 & 177 & 1298 & - & - & - & - & 316 & 1985 & $1.680 \mathrm{e}+03$ & 316 & 1985 & $1.450 \mathrm{e}+03$ & 177 & 1298 & $1.341 \mathrm{e}+03$ \\
\hline 17 & 199 & 1661 & - & - & - & - & 355 & 2205 & $5.048 \mathrm{e}+04$ & 369 & 2201 & $4.075 \mathrm{e}+04$ & 199 & 1661 & $3.462 \mathrm{e}+03$ \\
\hline 18 & 216 & 1894 & 561 & 5203 & 493 & 5100 & $\begin{array}{l}427 \\
463\end{array}$ & $\begin{array}{l}2599 \\
3079\end{array}$ & $6.840 \mathrm{e}+04$ & 427 & 3079 & $5.897 \mathrm{e}+04$ & 427 & 3079 & $4.077 \mathrm{e}+04$ \\
\hline 19 & 246 & 2225 & - & - & - & - & $\begin{array}{l}567 \\
597 \\
615\end{array}$ & $\begin{array}{l}3432 \\
5067 \\
4660\end{array}$ & $8.280 \mathrm{e}+04$ & 584 & 4101 & $7.158 \mathrm{e}+04$ & 467 & 3337 & $6.685 \mathrm{e}+04$ \\
\hline 20 & 283 & 2794 & 703 & 7163 & 703 & 6460 & $\begin{array}{l}673 \\
680 \\
691\end{array}$ & $\begin{array}{l}4826 \\
4905 \\
4941\end{array}$ & $1.12428 \mathrm{e}+05$ & 691 & 4941 & $1.0012 \mathrm{e}+05$ & $\begin{array}{l}578 \\
615\end{array}$ & $\begin{array}{l}4306 \\
4660\end{array}$ & $7.333 \mathrm{e}+04$ \\
\hline
\end{tabular}

different sequences. The preliminary results indicate that proposed HMOBB-BC algorithm appears to be most efficient algorithm to generate OGRs for optical WDM systems and outperforms the existing algorithms.

In order to see the complexity of realizing the unequal channel spacing, the existing researches does not show the implementation of their algorithms in real optical WDM systems. So, in order for the algorithms to be of practical use, it is desired that the performance of the algorithms for higher order OGRs channels may be evaluated and may be used to provide unequal channel spacing in real optical WDM systems. 


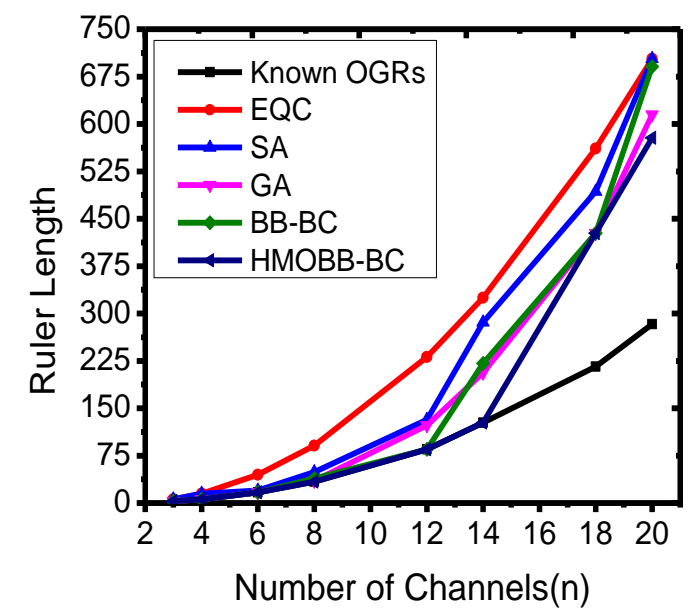

(a)

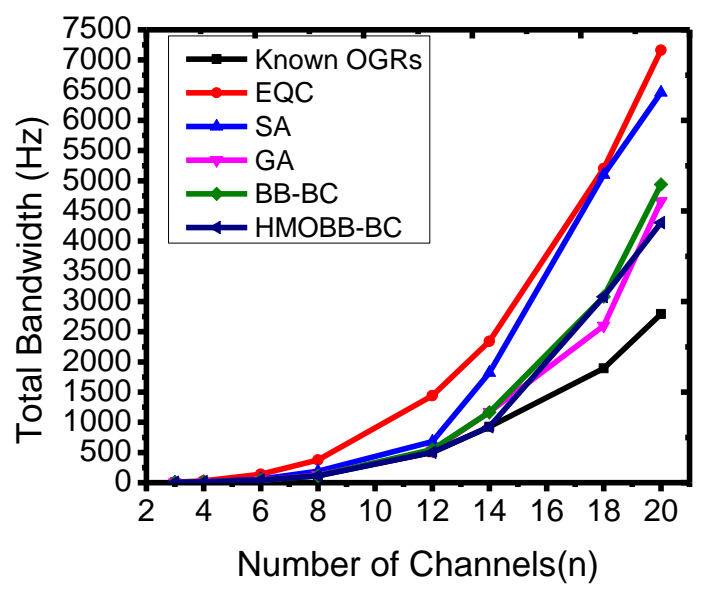

(b)

Fig 4: The proposed HMOBB-BC algorithm exhibits the significant reduction in (a) ruler length and (b) total occupied optical bandwidth in comparison to the existing algorithms i.e. Known OGR, EQC, SA, GA and BB-BC

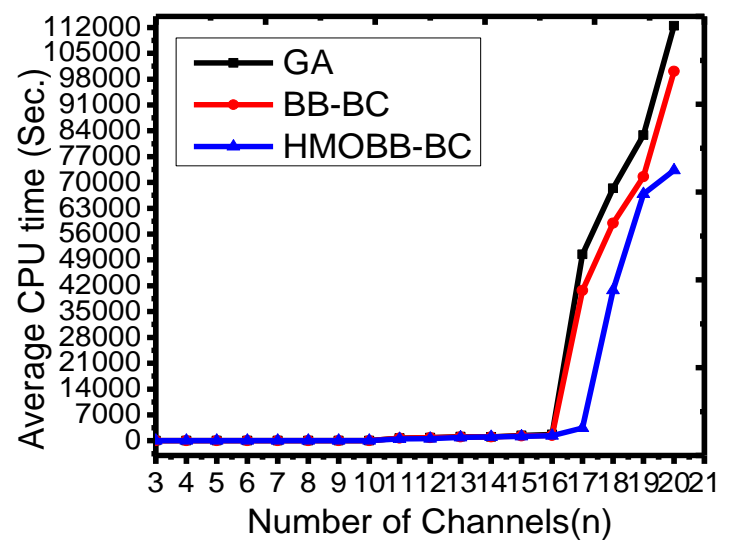

Fig 5: The proposed HMOBB-BC algorithm exhibits the significant reduction in average $\mathrm{CPU}$ time in sec. in comparison to the existing algorithms i.e. GA and BB-BC

\section{REFERENCES}

[1] Chraplyvy, A. R. 1990. Limitations on Lightwave Communications Imposed by Optical-Fiber
Nonlinearities, J. Lightwave Technology, Vol. 8, pp. 1548-1557.

[2] Kwong, W. C., and Yang, G. C. 1997. An Algebraic Approach to the Unequal-Spaced Channel-Allocation Problem in WDM Lightwave Systems, IEEE Transactions on Communications, Vol. 45, No.3, pp. 352-359.

[3] Saaid, N. M. 2010. Nonlinear Optical Effects Suppression Methods in WDM Systems with EDFAs: A Review, In Proceedings of the International Conference on Computer and Communication Engineering (ICCCE), Kuala Lumpur, Malaysia.

[4] Aggarwal, G. P. 2001. Nonlinear Fiber Optics, Edition, Academic Press, San Diego, CA.

[5] Thing, V. L. L., Shum, P., and Rao, M. K. (2004). Bandwidth-Efficient WDM Channel Allocation for Four-Wave Mixing-Effect Minimization, IEEE Transactions on Communications, Vol. 52, No. 12, pp. 2184-2189.

[6] Forghieri, F., Tkach, R. W., Chraplyvy, A. R., and Marcuse, D. 1994. Reduction of Four-Wave Mixing Crosstalk in WDM Systems Using Unequally Spaced Channels. IEEE Photonics Technology Letters, Vol. 6, No. 6, pp. 754-756.

[7] Babcock, W. C. 1953. Intermodulation interference in radio systems, Bell Systems Technical Journal, pp. 6373.

[8] Sardesai, H. P. 1999. A Simple Channel Plan to Reduce Effects of Nonlinearities In Dense WDM Systems. Lasers and Electro-Optics, (23-28, May-1999), pp. 183184.

[9] Forghieri, F., Tkach, R. W., and Chraplyvy, A. R. 1995. WDM systems with unequally spaced channels. J. Lightwave Technol., Vol. 13, pp. 889-897.

[10] Hwang, B. and Tonguz, O. K. 1998. A Generalized Suboptimum Unequally Spaced Channel Allocation Technique-Part I: In IM/DD WDM systems. IEEE Trans. Commun., Vol. 46, pp. 1027-1037.

[11] Tonguz, O. K. and Hwang B. 1998. A Generalized Suboptimum Unequally Spaced Channel Allocation Technique-Part II: In coherent WDM systems. IEEE Trans. Commun., Vol. 46, pp. 1186-1193.

[12] Atkinson, M. D., Santoro, N., and Urrutia, J. 1986. Integer sets with distinct sums and differences and carrier frequency assignments for nonlinear repeaters. IEEE Trans. Commun., Vol. COM-34.

[13] Randhawa, R., Sohal, J. S. and Kaler, R. S. 2009. Optimum Algorithm for WDM Channel Allocation for Reducing Four-Wave Mixing Effects. Optik120, pp. 898-904.

[14] http://www.compunity.org/events/pastevents/ewomp204/ jaillet_krajecki_pap_ew04.pdf.

[15] Bloom, G. S. and Golomb, S.W. 1977. Applications of Numbered Undirected Graphs. In Proceedings of the IEEE, Vol. 65, No. 4, pp. 562-570.

[16] Thing, V. L. L., Rao, M. K. and Shum, P. 2003. Fractional Optimal Golomb Ruler Based WDM Channel Allocation. In Proceedings of the 8th Opto-Electronics 
International Journal of Computer Applications (0975 8887) Volume 85 - No 9, January 201425and Communication Conference (OECC-2003), Vol. 23, pp. 631-632.

[17] Shearer, J. B. 1998. Some New Disjoint Golomb Rulers. IEEE Transactions on Information Theory, Vol. 44, No. 7, pp. 3151-3153.

[18] http://theinf1.informatik.unijena.de/teaching/ss10/oberse minar-ss10

[19] Robinson, J. P. 1979. Optimum Golomb Rulers. IEEE Transactions on Computers, Vol. C-28, No. 12, (December 1979), pp. 943-944.

[20] Shearer, J. B. 1990. Some New Optimum Golomb Rulers. IEEE Transactions on Information Theory. IT-36, pp. 183-184.

[21] Galinier, P., Jaumard, B., Morales, R. and Pesant, G. 2001. A constraint-Based Approach to the Golomb Ruler Problem. In Proceeding of 3rd International workshop on integration of AI and OR techniques (CP-AI-OR 2001).

[22] Leitao, T. 2004. Evolving the Maximum Segment Length of a Golomb Ruler. Genetic and Evolutionary Computation Conference, USA.

[23] Rankin, W. T. 1993. Optimal Golomb Rulers: An exhaustive parallel search implementation. M.S.thesis,DukeUniversity,Availableathttp://people.ee.d uke.edu/ wrankin/golomb/golomb.htm.

[24] Shobhika. 2005. Generation of Golomb Ruler Sequences and Optimization Using Genetic Algorithm. M.Tech. Thesis, Department of Electronics and Communication Engineering, Thapar Institute of Engineering and Technology, Deemed University, Patiala.

[25] Soliday, S. W., Homaifar, A. and Lebby, G. L. 1995. Genetic Algorithm Approach to the Search for Golomb Rulers. In Proceedings of the Sixth International Conference on Genetic Algorithms (ICGA-95), Morgan Kaufmann, pp. 528-535.

[26] Robinson, J. P. 2000. Genetic Search for Golomb Arrays. IEEE Transactions on Information Theory, Vol. 46, No. 3, pp. 1170-1173.

[27] Ayari, N., Luong, T. V. and Jemai, A. 2010. A Hybrid Genetic Algorithm for Golomb Ruler Problem. In Proceeding of ACS/IEEE International Conference on Computer Systems and Applications (AICCSA 2010), pp.1-4.

[28] Bansal, S., 2014. Optimal Golomb Ruler Sequence Generation for FWM Crosstalk Elimination: Soft Computing Versus Conventional Approaches. Applied Soft Computing Journal (Elsevier), Vol. 22, pp. 443-457.

[29] Bansal, S., Kumar, S., Sharma, H. and Bhalla, P. 2011. Generation of Golomb Ruler Sequences and Optimization Using Biogeography Based Optimization. In Proceedings of 5th International Multi Conference on Intelligent Systems, Sustainable, New and Renewable Energy Technology and Nanotechnology (IISN-2011), Institute of Science and Technology Klawad, Haryana, pp 282-288.

[30] Bansal, S., Kumar, S., Sharma, H. and Bhalla, P. 2011. Golomb Ruler Sequences Optimization: A BBO Approach. International Journal of Computer Science and Information Security (IJCSIS), Pittsburgh, PA, USA, Vol. 9, No. 5, pp. 63-71.

[31] Kumar S., Bansal S. and Bhalla P. 2012. Optimal Golomb Ruler Sequence Generation for FWM Crosstalk Elimination: A BB-BC Approach. In Proceedings of 6th International Multi Conference on Intelligent Systems, Sustainable, New and Renewable Energy Technology and Nanotechnology (IISN-2012), Institute of Science and Technology Klawad-133105, Haryana, India, pp. 255-262.

[32] Bansal S., Kumar S. and Bhalla P. 2013. A Novel Approach to WDM Channel Allocation: Big Bang-Big Crunch Optimization. In the proceeding of Zonal Seminar on Emerging Trends in Embedded System Technologies (ETECH-2013) organized by The Institution of Electronics and Telecommunication Engineers (IETE), Chandigarh Centre, Chandigarh, pp. 80-81.

[33] Bansal, S. and Singh, K., 2014. A Novel SoftComputing Algorithm for Channel Allocation in WDM Systems. International Journal of Computer Applications (IJCA), Vol. 85, No. 9, pp. 19-26.

[34] Bansal, S., Chauhan, R. and Kumar, P., 2014. A Cuckoo Search based WDM Channel Allocation Algorithm. International Journal of Computer Applications (IJCA), Vol. 96, No. 20, pp. 6-12.

[35] Jain, P., Bansal, S., Singh, A. K. and Gupta, N., 2015. Golomb Ruler Sequences Optimization for FWM Crosstalk Reduction: Multi-population Hybrid Flower Pollination Algorithm. Progress in Electromagnetics Research Symposium (PIERS), Prague, Czech Republic, pp. 2463-2467.

[36] Horn, J., Nafbliotis, N., and Goldberg, D. E. 1994. A Niched Pareto Genetic Algorithm for Multiobjective Optimization. Evolutionary Computation, 1994. IEEE World Congress on Computational Intelligence, Proceedings of the first IEEE Conference on, Vol. 1, pp 82-87.

[37] Yang, X.-S., Karamanoglu, M., and He., X. S. 2014. Flower Pollination Algorithm: A Novel Approach for Multi-objective Optimization. Engineering Optimization, Vol. 46, Issue 9, pp. 1222-1237, doi: $10.1080 / 0305215 \times .2013 .832237$.

[38] Dimitromanolakis, A. 2002. Analysis of the Golomb Ruler and the Sidon Set Problems, and Determination of Large, Near-Optimal Golomb Rulers. Master's Thesis, Department of Electronic and Computer Engineering, Technical University of Crete.

[39] Dollas, A., Rankin, W. T., and McCracken, D. 1998. A New Algorithm for Golomb Ruler Derivation and Proof of the 19 Mark Rulers. IEEE Transactions on Information Theory, Vol. 44, No. 1, pp. 379-382.

[40] Project OGR. http://www.distributed.net/OGR.

[41] Cotta, C., Dotu, I., Fernandez, Antonio J., and Hentenryck, Pascal V. 2007. Local Search-Based Hybrid Algorithms for Finding Golomb Rulers. Kluwer Academic Publishers, Boston, Vol. 12, Issue 3, pp. 263 291.

[42] http://mathworld.wolfram.com/PerfectRuler.html 
[43] http://mathworld.wolfram.com/GolombRuler.html

[44] Afshar, M. H., and Motaei, I. 2011. Constrained Big Bang-Big Crunch Algorithm For Optimal Solution of Large Scale Reservoir Operation Problem, International Journal of Optimization In Civil Engineering, pp. 357375 .

[45] Tabakov, P. Y. 2011. Big Bang-Big Crunch Optimization Method in Optimum Design of Complex Composite Laminates, World Academy of Science, Engineering and Technology, Vol. 77, pp. 835-839.

[46] Ahmadi, S. and Sedighizadeh, M. 2014. An Efficient Hybrid Big Bang-Big Crunch Algorithm for Reconfiguration of Distribution System for Loss Reduction, in Conference and exhibition on Electricity Distribution, Vol 14-E-aaa-0000.

[47] Erol, O. K. and Eksin, I. 2006. A New Optimization Method: Big Bang-Big Crunch. Advances in Engineering Software, Vol.37, pp. 106-111.

[48] Yesil, E. and Urbas, L. 2010. Big Bang-Big Crunch Learning Method for Fuzzy Cognitive Maps, World Academy of Science, Engineering and Technology 71, pp. 815-824.

[49] Zandi, Z., Afjei, E., and M. Sedighizadeh. Hybrid Big Bang-Big Crunch Optimization Based Optimal Reactive Power Dispatch for Voltage Stability Enhancement. In
Electrical and Computer Engineering Department, Shahid Beheshti University, G.C., Velenjak, Tehran, Iran, Vol. 47, No.2, pp. 537-546.

[50] Goldberg, D. E. 1989. Genetic Algorithms in Search, Optimization, and Machine Learning. Addison Wesley, USA.

[51] Price, K., Storn, R. and Lampinen. J. 2005. Differential Evolution-A Practical Approach to Global Optimization. Springer, Berlin, Germany.

[52] Storn, R., and Price, K. V. 1997. Differential Evolution-A Simple and Efficient Heuristic for Global Optimization Over Continuous Spaces. Journal of Global Optimization, Vol. 11, No. 4, pp. 341-359.

[53] http://in.mathworks.com/help/matlab/index.html.

[54] Shearer, J. B., 2001. Golomb Ruler Table. Mathematics Department, IBM Research. Available at http://www.research.ibm.com/people/s/shearer/grtab.htm 1.

[55] Colannino, J. 2003. Circular and Modular Golomb Rulers.

[56] Shearer, J. B. Smallest Known Golomb Rulers. Mathematics Department, IBM Research. Available at http://www.research.ibm.com/people/s/shearer/gropt.htm 1 